Revista Iberoamericana de Ciencias de la Actividad Física y el Deporte

\title{
ABSTRACTS DEL I CONGRESO INTERNACIONAL EN CIENCIAS DEL EJERCICIO Y LA ACTIVIDAD FÍSICA. SAN JOSÉ DE COSTA RICA. AGOSTO DE 2017.
}

ABSTRACTS FROM THE I INTERNATIONAL CONGRESS ON SCIENCE OF EXERCISE AND PHYSICAL ACTIVITY. SAN JOSÉ DE COSTA RICA. AUGUST 2017.

SUPLEMENTOS NUTRICIONALES PARA EL AUMENTO DE LA MASA MUSCULAR Y LA PÉRDIDA DE GRASA

NUTRITIONAL SUPPLEMENTS FOR MUSCLE BUILDING AND FAT LOSS

Bonilla, DA ${ }^{1,2,6, *}$; Pérez-Idárraga, A $^{3,6}$; Marín, E,6; Kammerer, ${ }^{5}{ }^{5}$; Petro, JL ${ }^{2,6}$. 1 Departamento de Bioquímica y Biología Molecular, Universidad Distrital Francisco José de Caldas, Bogotá D.C., Colombia.

${ }^{2}$ Grupo de Investigación en Ciencias de la Actividad Física, el Deporte y la Salud, Universidad de Córdoba, Montería, Colombia.

${ }^{3}$ Posgrado de Medicina Aplicada a la actividad física y el deporte, Universidad de Antioquia, Medellín, Colombia.

4 Laboratorio de Biología Molecular, Centro de Investigaciones EndocrinoMetabólicas Dr. Félix Gómez, Universidad del Zulia, Maracaibo, Venezuela 5 Área Cineantropometría - Centro de Estudios, Facultad de Ciencias de la Nutrición y los Alimentos, Universidad CES, Medellín, Colombia.

${ }^{6}$ Research Team, DB SPORT SCIENCES, DBSS. LA ACTIVIDAD FÍSICA. SAN JOSÉ DE COSTA RICA. AGOSTO DE 2017. 
Correspondencia: Diego A. Bonilla Ocampo. Miembro investigador en Laboratorio de Bioquímica y Biología Molecular de la Universidad Distrital Francisco José de Caldas, Investigador GICAFS y Director DB SPORT SCIENCES. Bogotá, Colombia. direccion@dbsportsciences.com $+573203352050$

\section{RESUMEN}

Adquirir una buena forma física es el objetivo de la mayoría de las personas que asisten a un centro de acondicionamiento físico; sin embargo, este proceso requiere de una individualización en la programación del entrenamiento y en la estrategia de alimentación, razón por la cual muchas personas no alcanzan sus metas y resultan adoptando prácticas innecesarias y peligrosas de suplementación. Teniendo en cuenta lo anterior, el consumo de suplementos nutricionales solamente debe contemplarse posterior a un correcto balance energético, distribución de macronutrientes y timing nutricional, acorde al objetivo del individuo y bajo el asesoramiento de un profesional, para permitir así una correcta alfabetización nutricional. De acuerdo a la evidencia científica actual cuando el objetivo es incrementar la masa muscular, y solo de recomendarse por un profesional, se podría hacer uso de la suplementación con proteína de suero de leche o Whey Protein ( $0.4-0.5 \mathrm{~g} / \mathrm{kg} /$ post-ejercicio), caseína ( $\approx 30 \mathrm{~g}$ antes de dormir) y monohidrato de creatina $(0.3 \mathrm{~g} / \mathrm{kg} /$ día durante 5-7 días seguido de $0.075 \mathrm{~g} / \mathrm{kg}$ / post-entrenamiento durante $4-6$ semanas). Se requiere más investigación respecto al consumo de Ashwagandha - Withania somnifera (300 mg dos veces al día) durante el mesociclo de entrenamiento de la fuerza. Por otro lado, durante una estrategia de reducción de grasa corporal para sujetos con sobrepeso u obesidad, los profesionales de la salud que realizan el seguimiento del caso pueden valorar el consumo de un reemplazo de comida en polvo (Meal Replacement Powder), como una alternativa para incrementar el aporte diario de proteína si se requiere, además de contemplar el consumo de Isomaltulosa (Palatinosa ${ }^{\circledR}$ ) o amilopectinas modificadas en algunas comidas 0 1-2 horas antes del ejercicio, sobre todo para controlar la hiperinsulinemia 
postprandial. La ingesta de un suplemento de cafeína anhidra $\left(2-4 \mathrm{mg} \cdot \mathrm{kg}^{-1} \cdot \mathrm{dí}^{-}\right.$ $\left.{ }^{1}\right)$ y algunos compuestos termogénicos; tales como, capsaicina (capsicum), té verde (Camelia sinensis), pimienta negra (Piper nigrum) y jengibre (Ginger officinale) pueden ser una alternativa para mejorar la adherencia a la dieta y aprovechar su potencial para reducir el porcentaje de grasa corporal, aunque se necesita más investigación a largo plazo para respaldar un efecto con significancia clínica.

PALABRAS CLAVE: proteína de suero, monohidrato de creatina, cafeína, capsicum, masa magra, composición corporal

\begin{tabular}{lllll}
\hline ENTRENAMIENTO & DE & LA & FUERZA. ASPECTOS
\end{tabular} FISIOLÓGICOS Y METODOLÓGICOS

RESISTANCE TRAINING PHYSIOLOGICAL AND METHODOLOGICAL ASPECTS

Petro, JL ${ }^{1, *}$; Bonilla $\mathrm{DA}^{1,2, ;}$ Marín, $\mathrm{E}^{3}$; Vargas, $\mathrm{S}^{4}$.

${ }^{1}$ Grupo de Investigación en Ciencias de la Actividad Física, el Deporte y la Salud, Universidad de Córdoba, Montería, Colombia. ilpetros@hotmail.com jorgelpetro@correo.unicordoba.edu.co

${ }^{2}$ Departamento de Bioquímica y Biología Molecular, Universidad Distrital Francisco José de Caldas, Bogotá D.C., Colombia.

${ }^{3}$ Laboratorio de Biología Molecular, Centro de Investigaciones EndocrinoMetabólicas Dr. Félix Gómez, Universidad del Zulia, Maracaibo, Venezuela ${ }^{4}$ EADE-University of Wales, Málaga, Spain.

* Correspondencia: Jorge Luis Petro-Soto. Grupo de Investigación en Ciencias de la Actividad Física, el Deporte y la Salud, Universidad de Córdoba, Montería, Colombia. LA ACTIVIDAD FÍSICA. SAN JOSÉ DE COSTA RICA. AGOSTO DE 2017. 


\section{RESUMEN}

Un buen estatus estructural (desarrollo muscular) y funcional (niveles de fuerza) del musculo esquelético (ME) constituye un pilar fundamental para la salud. El papel del ME, a luz del conocimiento actual, va más allá de la mera locomoción, destacándose la acción secretora (autocrina, paracrina y endocrina) y la comunicación cruzada que tiene con otros tejidos (e.g adiposo, nervioso, óseo) a través de las mioquinas, que son un conjunto de citocinas producidas y secretadas por el ME. Estas mioquinas, inducidas por el ejercicio físico, son mediadoras en las respuestas o adaptaciones al ejercicio físico, como es la mejora en la captación de glucosa, incremento en la oxidación de las grasas, regulación de la regeneración muscular, aumento de la termogénesis y, adicionalmente, pueden equilibrar o contrarrestar los efectos proinflamatorios causados por la sobreproducción de ciertas adipoquinas. De esta manera, independientemente a los propósitos particulares que se buscan con el entrenamiento fuerza (RT), tener una adecuada cantidad y calidad de masa muscular debe ser un objetivo de toda la población. Sin embargo, factores como el envejecimiento, el comportamiento sedentario, la inactividad física y la alimentación inadecuada, conducen a alteraciones en el recambio proteico muscular, es decir, disminución en la síntesis de proteína muscular e incremento en la degradación de las mismas. Teniendo en cuenta lo anterior, se incrementa el riesgo de sarcopenia y, por consiguiente, efectos adversos como discapacidad física, calidad de vida deficiente y mortalidad. Ante este escenario, el RT constituye una estrategia de intervención que, en conjunto con la nutrición, juega un rol importante en la prevención y tratamiento de entidades complejas como la sarcopenia y la obesidad. No obstante, para optimizar los efectos del RT y minimizar los riesgos de lesión, es necesario que se tengan en cuenta aspectos metodológicos de la planificación, como es la adherencia a los programas de acondicionamiento físico, componentes de la carga (e.g. volumen, intensidad, frecuencia), la progresión, selección de ejercicio, entre otras variables. En conclusión, el RT es un componente fundamental dentro de los programas de acondicionamiento físico, no siendo exclusivo para incrementar el rendimiento deportivo, sino también para la salud y la calidad de vida; adicionalmente, los 
especialistas en ciencias del ejercicio, tiene la responsabilidad de educar a las personas para que adopten hábitos y estilos de vida saludables que, en general, contemplen la práctica regular de actividad física $\mathrm{y}$, específicamente, la realización de RT.

PALABRAS CLAVES: Aptitud física, ejercicio, mioquinas, músculo.

\section{NUTRICIÓN EN FITNESS Y DEPORTES DE FUERZA}

\section{FITNESS AND STRENGHT SPORTS NUTRITION}

\section{Solís-Agüero, ML $^{1}$}

${ }^{1}$ Licenciada en Nutrición \& DBSS Nutrition Coordinator, San José, Costa Rica. info@krishafit.com

\section{RESUMEN}

Los deportes de fuerza son evaluados por el rendimiento, fuerza y velocidad del atleta, razón por la cual la preocupación principal de estos es mantener una ingesta diaria adecuada de calorías. El peso corporal juega un papel importante, ya sea para aumento de masa muscular, como en la halterofilia, o el caso contrario de la gimnasia, donde se busca minimizar la masa corporal pero al mismo tiempo aumentar la fuerza y la potencia. Por otro lado, en el fisicoculturismo y fitness los atletas son evaluados por su apariencia estética. En este último, la meta final de todo atleta es presentar un cuerpo musculado, simétrico, bien proporcionado y con niveles bajos de grasa corporal, sometiéndose a regímenes estrictos de alimentación. Para optimizar la ganancia de masa muscular y reducir el porcentaje de grasa corporal, los atletas obtendrán mejores resultados si realizan un acercamiento nutricional basado en evidencia científica actualizada. En las estrategias de alimentación en deportes de fuerza, la ingesta de alimentos y líquidos es más manejable en comparación con otros deportes, debido a su naturaleza intermitente. Así, la planeación de los alimentos 
a ingerir antes y después de la competición es crucial para que los atletas puedan recuperarse entre cada ejecución/combate o presentación. Ahora bien, la preparación para la competición en el área fitness puede dividirse en tres fases; fuera de temporada, de transición y pre-competitivo. En la primera fase, el objetivo es el anabolismo muscular mediante un balance positivo de energía; la segunda fase se caracteriza por un déficit calórico que propicie la pérdida paulatina de grasa corporal, mediante una disminución progresiva de los carbohidratos (de 6-10 $\mathrm{g} \cdot \mathrm{kg}^{-1} \cdot \mathrm{d}^{-1}$ a 2-3 $\mathrm{g} \cdot \mathrm{kg}^{-1} \cdot \mathrm{d}^{-1}$ ); y finalmente una fase que promueve la reducción excesiva de grasa corporal, aumentando la ingesta de proteína diaria por encima de los $3 \mathrm{~g} \cdot \mathrm{kg}^{-1} \cdot \mathrm{d}^{-1}$ con la finalidad de proveer una cantidad adecuada de aminoácidos para la síntesis de proteína y evitar la pérdida de masa muscular. En ésta última fase se realiza un ajuste constante y progresivo de las calorías y macronutrientes totales dependiendo de la apariencia física del atleta. En conclusión, la alimentación debe ser adaptada para apoyar el cambio de los objetivos individuales, los niveles de entrenamiento y los requisitos a lo largo de la temporada y el ciclo competitivo.

PALABRAS CLAVE: Proteínas, Fuerza, Hipertrofia, Rendimiento Atlético, Dieta

\section{NUTRICION PARA DEPORTES DE LARGA DURACIÓN}

\section{NUTRITION IN FITNESS AND STRENGTH SPORTS}

\section{Gómez-Martín, LA ${ }^{1}$}

${ }^{1}$ Licenciado en Nutrición, SNT Center (Sport Nutrition and Trainning Center), Jalisco, México.gomezluisnutricion@hotmail.com

\section{RESUMEN}

Las adaptaciones buscadas con el entrenamiento programado de la resistencia a través de un periodo de tiempo consisten principalmente en el aumento del consumo máximo de oxígeno, el mejoramiento del umbral 
ventilatorio, mayor almacenamiento de glucógeno muscular y hepático, mayor biogénesis y densidad mitocondrial, entre otras. No obstante, para que dichas adaptaciones ocurran se requiere que el aporte dietético de cada macronutriente se establezca según la etapa en la que se encuentre el atleta, bien sea preparatoria para competición, competitiva o fuera de temporada. Se recomienda que los ajustes nutricionales se establezcan en relación de gramos de macronutriente por kilogramos totales de masa corporal. Respecto al aporte de los mismos, se aconseja entre $7-10 \mathrm{~g} \mathrm{CHO} \cdot \mathrm{kg}^{-1} \cdot \mathrm{dí}^{-1}$, de 1 a $2 \mathrm{~g}$ de grasa por $\mathrm{kg}$ al día y entre $1.4-1.6 \mathrm{~g} \mathrm{PRO} \cdot \mathrm{kg}^{-1} \cdot \mathrm{di}^{-1}$. Ahora bien, es importante considerar ingestas en el periodo peri-entrenamiento, en donde se siguen pautas específicas para asegurar las máximas reservas de glucógeno previo al entrenamiento y/o competición, además de asegurar una adecuada hidratación, aporte de electrolitos $\left(\mathrm{Na}^{+}, \mathrm{K}^{+}\right.$, etc.), consumo de mezclas de carbohidratos que proporcionen un flujo constante de sustratos energéticos durante la competencia y una óptima recuperación del sistema musculo-esquelético entre sesiones de entrenamiento o competencia. Las ayudas ergogénicas nutricionales en los deportes de larga duración son aquellos compuestos que mejoran los procesos de adaptación y aumentan el rendimiento durante la prueba, tales como; bebidas deportivas, geles o gomitas con carbohidratos, proteínas, electrolitos, cafeína, bicarbonato de sodio y nitratos. A manera de conclusión, si se logra diseñar un plan de alimentación que tome en cuenta la periodización y planificación del entrenamiento, los requerimientos específicos de macronutrientes, un adecuado aporte de micronutrientes, un correcto manejo de las estrategias nutricionales en el periodo peri-entrenamiento $y$, de ser necesario, el consumo de ayudas ergogénicas nutricionales, se tendrá una mayor probabilidad de tener un máximo rendimiento durante las competencias, al soportar la carga de trabajo durante los entrenamientos y lograr una recuperación integral tras los mismos.

PALABRAS ClAVE: Dietética, Resistencia Física, Rendimiento Deportivo, Suplementación. 
DETECCIÓN DE POSIBLES TALENTOS DEPORTIVOS, EN EL ENTORNO ESCOLAR

DETECTION OF POSSIBLE SPORT TALENTS, IN THE SCHOOL ENVIRONMENT

\begin{abstract}
Alexander-Cortez, PA $^{\mathbf{1}}$
${ }^{1}$ MSc. Fisiología del Ejercicio. Universidad Nacional Experimental Politécnica (UNEXPO), Lara, Venezuela. p alexander ve@yahoo.com
\end{abstract}

\title{
RESUMEN
}

Los programas para la detección de posibles talentos deportivos, por las características del grupo objetivo tiene su máxima expresión de aplicación en el contexto escolar. Las acciones conexas con la evaluación cuantitativa de variables relacionadas con la salud y la aptitud, pueden extenderse hacia áreas que potencien el deporte de competencia. Revisando el programa mundial de las competencias deportivas y los resultados alcanzados en las mismas, se puede inferir que el desempeño global de los atletas cada día mejora, disminuyendo a la vez la edad a la cual alcanzan el nivel máximo de desempeño. Quizás este fenómeno se deba a la introducción de procedimientos para la detección temprana de los futuros atletas y a la aplicación de métodos de entrenamiento más efectivos. El modelo para la detección de talentos que se propone cumple las etapas señaladas en la mayoría de los trabajos que, en este sentido, se conocen. El mismo involucró la construcción de las normas nacionales para niños y jóvenes entre 7 y 18 años de edad (en el presente caso $\mathrm{n}=7.044 ; 11$ grupos de edad por género), aplicando una batería integrada por el peso, la talla, conjuntamente con las pruebas de $1200 \mathrm{~m}, 30 \mathrm{~m}$ velocidad, salto vertical, potencia, flexibilidad, abdominales y lanzamiento de pelota de béisbol. A partir del grupo de pruebas, se generó un modelo lógico de asociación entre ellas y siete actividades deportivas generales (resistencia, resistencia media, 
potencia, velocidad, salto en longitud y salto en proyección vertical), conjuntamente con un modelo matemático basado en la distribución percentil de los datos en cada una de las pruebas que componen la batería, para establecer, con el rango deseado de aproximación, el potencial de cada niño o joven en el contexto de los requisitos de cada una de las especialidades deportivas consideradas. De esta manera, es posible aplicar la siguiente ecuación: $\mathbf{X}=$ $\left(\mathrm{Y}_{1} * \mathrm{P}_{1}\right)+\left(\mathrm{Y}_{2} * \mathrm{P}_{2}\right) \ldots+\left(\mathrm{Y}_{n} * \mathrm{P}_{n}\right)$, donde $\mathrm{X}=$ es el potencial de dotes para una especialidad deportiva determinada; $\mathbf{Y}=$ es el baremo de importancia y $\mathbf{P}=$ el percentil alcanzado en la distribución de una prueba determinada.

PALABRAS CLAVE: Detección de Talentos, Atleta, Normas Nacionales, Batería de Pruebas, Modelo Matemático

ESTRATEGIAS DE TRABAJO PARA LA REDUCCIÓN DEL TEJIDO ADIPOSO

TRAINING STRATEGIES FOR THE REDUCTION OF ADIPOSE TISSUE

Vargas, $\mathrm{S}^{1,5}$; Petro, JL ${ }^{2,5}$; Bonilla DA ${ }^{2,3,5}$; Carbone, $\mathrm{L}^{4}$

${ }^{1}$ EADE-University of Wales, Málaga, Spain

${ }^{2}$ Grupo de Investigación en Ciencias de la Actividad Física, el Deporte y la Salud, Universidad de Córdoba, Montería, Colombia.

${ }^{3}$ Departamento de Bioquímica y Biología Molecular, Universidad Distrital Francisco José de Caldas, Bogotá D.C., Colombia.

${ }^{4}$ Physical Activity and Sports, Faculty of Education Sciences and Social Communication, University of Salvador, Buenos Aires, Argentina;

${ }^{5}$ Research Team, DB SPORT SCIENCES, DBSS.

Correspondencia: Salvador Vargas Molina. Grupo de Investigación en Ciencias de la Actividad Física, EADE-Málaga. Centro Adscrito University of Wales salvadorvargasmolina@gmail.com; salvadorvargas@eade.es LA ACTIVIDAD FÍSICA. SAN JOSÉ DE COSTA RICA. AGOSTO DE 2017. 


\section{RESUMEN}

En el fitness actual y con un objetivo estético se utilizan diversas estrategias de entrenamiento con el propósito de reducir el porcentaje de tejido adiposo (TA). La falta de preparación de un gran número de entrenadores, unido al vacío en la legislación vigente en la regulación profesional de especialistas en ciencias del ejercicio y sus diferentes competencias, hacen necesarias herramientas que optimicen los resultados en sujetos que persiguen una mejora en su composición corporal. Por ello, se considera oportuno establecer unas pautas sobre las diferentes estrategias para reducir el tejido adiposo. La primera opción consiste en organizar las variables de programación del entrenamiento de fuerza-hipertrofia acorde a la reducción de grasa, conjuntamente con la estrategia nutricional y controlada por un especialista en nutrición deportiva, donde primará el déficit calórico. Si fuese necesario utilizar opciones de entrenamiento cardiovascular, se podría optar por la utilización de entrenamientos de alta intensidad (HIIT) con diferentes medios. Por otro lado, contemplar la utilización de sesiones de aeróbico extensivo (AE) y de entrenamiento cardiovascular en ayunas (CAY) como posibles alternativas. Finalmente, las cardio-aceleraciones (CA) podrían considerarse como una opción alternativa, debido a que propicia sesiones que intercambiarían estímulos de fuerza y de resistencia aeróbica. La utilización de una u otra estrategia dependerá de la composición corporal del sujeto, del tiempo que falte para el pico competitivo, de la capacidad de respuesta y de la disponibilidad de tiempo en el microciclo de entrenamiento.

PALABRAS CLAVES: Fitness, HIIT, Entrenamiento de Fuerza, Hipertrofia Muscular 